\title{
A high precision semi-analytic mass function
}

To cite this article: Antonino Del Popolo et al JCAP03(2017)032

\section{Related content}

A unified solution to the small scale problems of the CDM model II: introducing parent-satellite interaction

A. Del Popolo and M. Le Delliou

Computation of the halo mass function using physical collapse parameters: application to non-standard cosmologies I. Achitouv, C. Wagner, J. Weller et al.

View the article online for updates and enhancements.

- $\frac{\text { THE HALO MASS FUNCTION FROM }}{\text { EXCURSION SET THEORY. II. }}$ Michele Maggiore and Antonio Riotto

\section{Recent citations}

- Mass-temperature relation in CDM and
$\frac{\text { modified gravity }}{\text { Antonino Del Popolo et al }}$
- Correlations in the matter distribution in
ALASH galaxy clusters
Antonino Del Popolo et al
- Dark compact objects: An extensive
overview
Maksym Deliyergiyev et al

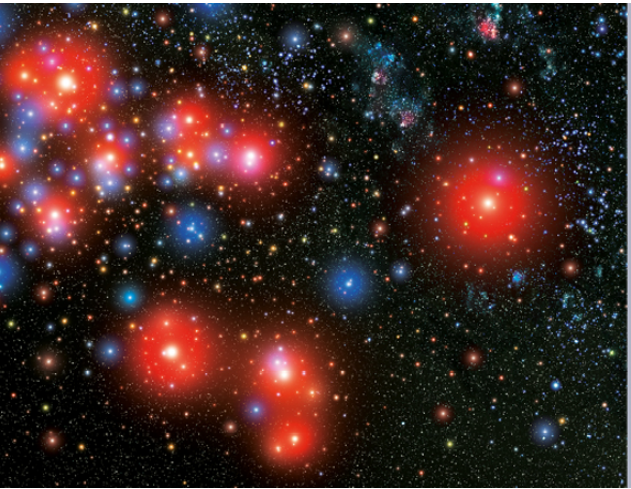

\section{A.A IOP Astronomy ebooks}

Part of your publishing universe and your first choice for astronomy, astrophysics, solar physics and planetary science ebooks. iopscience.org/books/aas 


\title{
A high precision semi-analytic mass function
}

\author{
Antonino Del Popolo, ${ }^{a, b, c}$ Francesco Pace $^{d}$ and \\ Morgan Le Delliou ${ }^{e, f, g}$ \\ ${ }^{a}$ Dipartimento di Fisica e Astronomia, University of Catania, \\ Viale Andrea Doria 6, I-95125 Catania, Italy \\ ${ }^{b}$ INFN sezione di Catania, \\ Via S. Sofia 64, I-95123 Catania, Italy \\ ${ }^{c}$ International Institute of Physics, Universidade Federal do Rio Grande do Norte, \\ 59012-970 Natal, Brazil \\ ${ }^{d}$ Jodrell Bank Centre for Astrophysics, School of Physics and Astronomy, \\ The University of Manchester, \\ Manchester, M13 9PL, U.K. \\ ${ }^{e}$ Instituto de Física Teorica, Universidade Estadual de São Paulo (IFT-UNESP), \\ Rua Dr. Bento Teobaldo Ferraz 271, Bloco 2 - Barra Funda, \\ 01140-070 São Paulo, SP Brazil \\ ${ }^{f}$ Institute of Theoretical Physics, Physics Department, Lanzhou University, \\ No. 222, South Tianshui Road, Lanzhou, Gansu 730000, P.R. China \\ ${ }^{g}$ Instituto de Astrofísica e Ciências do Espaço, \\ Universidade de Lisboa, Faculdade de Ciências, \\ Ed. C8, Campo Grande, 1769-016 Lisboa, Portugal \\ E-mail: adelpopolo@oact.inaf.it, francesco.pace@manchester.ac.uk, \\ delliou@ift.unesp.br
}

Received August 24, 2016

Revised February 8, 2017

Accepted March 3, 2017

Published March 14, 2017

\begin{abstract}
In this paper, extending past works of Del Popolo, we show how a high precision mass function (MF) can be obtained using the excursion set approach and an improved barrier taking implicitly into account a non-zero cosmological constant, the angular momentum acquired by tidal interaction of proto-structures and dynamical friction. In the case of the $\Lambda \mathrm{CDM}$ paradigm, we find that our MF is in agreement at the $3 \%$ level to Klypin's Bolshoi simulation, in the mass range $M_{\text {vir }}=5 \times 10^{9} h^{-1} M_{\odot}-5 \times 10^{14} h^{-1} M_{\odot}$ and redshift range $0 \lesssim z \lesssim 10$. For $z=0$ we also compared our MF to several fitting formulae, and found in particular agreement with Bhattacharya's within $3 \%$ in the mass range $10^{12}-10^{16} h^{-1} M_{\odot}$. Moreover, we discuss our MF validity for different cosmologies.
\end{abstract}

Keywords: galaxy formation, dark matter theory 


\section{Contents}

1 Introduction $\quad 1$

2 ESF choices of barrier $\quad 4$

3 Multiplicity and mass function $\quad 7$

4 Results $r$

5 Discussion $\quad 15$

$\begin{array}{lr}\text { A Inclusion of the angular momentum } & 16\end{array}$

$\begin{array}{lr}\text { B Expressions for the mass function } & 18\end{array}$

\section{Introduction}

The $\Lambda$ CDM model, often referred to as "cosmic concordance" model or standard model of Big Bang cosmology, is a "double dark" cosmological model, in which the Universe is constituted by cold dark matter plus a vacuum density energy, represented by the cosmological constant $\Lambda$. On large and intermediate scales this model has been proven to be very successful in fitting a large variety of data $[1-8] .^{1}$

A further fundamental test of the $\Lambda \mathrm{CDM}$ model resides in the accurate prediction of the halo mass function (MF), namely the mass distribution of dark matter halos, or more in detail the number density of dark matter halos per mass interval [see 2, 18, 19].

At redshifts $z \leq 2$, the high mass end of the MF (clusters of galaxies) is very sensitive to cosmological parameters like $\sigma_{8}$, variations in cosmological parameters like the Universe matter and dark energy (DE) content $\left(\Omega_{\mathrm{m}}\right.$ and $\left.\Omega_{\Lambda}\right)$, the equation of state parameter $w$ and its evolution $[20-29] .^{2}$

At higher redshifts, the MF is an important probe of the Universe reionization history [e.g. 30] and quasar abundance [e.g. 31].

Apart from its use to determine the cosmological parameters, the MF is a fundamental ingredient to study DM distribution, aspects of formation and evolution of galaxies through semi-analytic and analytic models. Furthermore, a high precision MF is related to ongoing and upcoming surveys detecting clusters using optical observations, X-rays, or the SunyaevZel'dovich (SZ) effect. Thus, clearly, a simple and accurate high precision MF, valid for different cosmologies and redshifts and allowing a precise extraction of cosmological parameters is a helpful and valuable asset.

[32] (PS) proposed a simple model in which initial fluctuations are spherical, with a Gaussian distribution, and their evolution is followed from the linear phase until collapse using a spherical collapse model (SCM). At the virialization epoch (identified with the collapse

\footnotetext{
${ }^{1}$ From a theoretical point of view, the model is afflicted by the fine tuning problem $[9,10]$ and the cosmic coincidence problem. At kpc-scales, the $\Lambda \mathrm{CDM}$ model is suffering other problems: the cusp/core problem [11$16]$ or the missing satellite problem $[7,17]$.

${ }^{2} \sigma_{8}$ represents the linear power spectrum amplitude on a scale of $8 h^{-1} \mathrm{Mpc}$.
} 
redshift), the density contrast, $\delta=\frac{\rho-\bar{\rho}}{\bar{\rho}}$, calculated within linear perturbation theory, gets the value $\delta_{\mathrm{c}} \simeq 1.686$ for an Einstein-de Sitter cosmology. Under the assumption that the density field has a Gaussian probability distribution, one can calculate the probability that the overdensity on a given scale exceeds the critical value $\delta_{\mathrm{c}}$, which is independent from the mass of the collapsing object. This quantity is proportional to the number of cosmic structures characterized by a density perturbation greater than $\delta_{c}$. Unfortunately, in the PS theory the number of objects in the high mass tail is underpredicted, and conversely for objects in the low mass tail of the MF [e.g. 33-36]. Even the extended-PS formalism taking merging into account [37-40] does not solve the problem.

As shown in [41] (eq. 28, figure 6), the tidal interaction with neighbours and the angular momentum acquired modifies the collapse of a given region. As a result $\delta_{\mathrm{c}}$ depends on mass and this changes the mass function $[42,43]$.

Similarly, [44] showed that moving from a spherical to an elliptical geometry, the collapse depends on the initial overdensity and shear, and $\delta_{\mathrm{c}}$ depends on mass. The mass function obtained with the elliptical collapse was shown to be in agreement with N-body simulations [45] (ST). However, a deeper analysis of those semi-analytic models for the mass function showed some problems: the PS MF, as already reported, overpredicts the MF at all high and medium masses [46], and even the ST MF overpredicts the halo number at large masses [47]. The situation worsens if one studies the PS and ST MF evolution. Simulations have been used to obtain a better understanding of the MF at low redshifts and of its evolution at high redshifts. [35] tested the ST MF up to $z=5$ and down to $\simeq 3 \times 10^{11} M_{\odot}$, finding discrepancies only at uncommon (rare) density enhancements. [46, 48] found that the PS MF underestimates the rarest haloes in their simulations by a factor of $\simeq 5$, namely proto-galaxies at $z=30$, and galaxies with masses $\simeq 10^{11} h^{-1} M_{\odot}$ at $z=10$. Haloes hosting star populations at $z=30$ were underestimated by a factor of $\simeq 2$. The ST MF had a much better performance than the PS MF, but its predictive power decreased with increasing masses and redshifts. Their overestimation goes up to a factor of $\simeq 3$ for the rarest haloes [47, 48]. In the case of the Bolshoi simulation [49], the discrepancy is smaller than $10 \%$ at $z=0$ in the mass range $5 \times 10^{9}-5 \times 10^{14} M_{\odot}$, while at $z=10$ the $\mathrm{ST} \mathrm{MF}$ gives $\simeq 10$ times more haloes than simulations. [50] compared several MF [32, 44, 46, 48, 51-55] with their Friends-of-Friends (FoF) MF (see the following) at redshift $z=0$. Excluding the PS MF, the remaining agree to $\simeq 10 \%$ for masses $\leq 10^{15} M_{\odot}$.

The examples above show how an imprecise MF produces incorrect predictions, especially for halo numbers at high redshift (e.g., the number density of high redshift QSOs) or for astrophysical phenomena happening at high redshift (reionization scenarios and reionization history).

The universality of the MF, that is its independence on cosmology and redshift, poses an important issue studied by several authors [e.g. 48, 50, 52-54, 56]. Obviously a universal MF would avoid the need to use N-body simulations to study it for different cosmologies and for its time evolution.

The majority of numerical simulations identify halos by one of two different techniques: either friends-of-friends (FOF) or spherical overdensity (SO) algorithms. The FOF method identifies halos by a percolation technique, connecting particles, within a certain distance (the linking length b) to each other, in the same halo. The linking length is typically chosen between $b=0.15$ and $b=0.2$, where $b$ is defined with respect to the mean interparticle spacing. The FOF halo mass function scales very close to the "universal" behaviour [49].

The SO method first finds the halo centre from potential minimum or most bound particle to identify haloes with spheres reaching a threshold density, given with respect to 
either critical or background density. Typical ratios with respect to the critical density $\rho_{\mathrm{c}}$ are chosen from $\Delta=200$ to 500 (or higher for clusters). SO-based halo mass functions are not universal especially at higher $z$.

The resulting two mass functions at $z=0$ display close similarity for lower mass haloes, while the FOF case yields greater MF in higher-mass haloes [50]. At higher redshifts, the SO MF falls to $\sim 80 \%$ of the FOF at lower masses, with a stronger marked decrease at higher masses [50].

The disagreement between FOF and SO is likely related to the tendency of FOF to link structures before they become a part of a virialized halo, a feature more frequent with the rare most massive halos, that tend to be out of equilibrium and in the process of merging. As a result, FOF masses are artificially inflated.

However, for isolated, relaxed halos, SO and FOF masses are strongly correlated [56, 57]. In cosmological simulations the mapping between the two halo definitions can be considered one-to-one at the $5 \%$ level of accuracy [58]. Nonetheless, a fair fraction of halos in simulations are irregular: currently favoured cosmologies yield $15 \%-20 \%$ of FOF halos with irregular substructure with linking length $b=0.2$ or two or more major halo components linked together [58]. Such irregular halos not only fail to follow the simple SO to FOF mapping, but also lack a clear definition for halo mass.

Most numerical simulations have used FoF masses with linking length $b=0.2$. As several authors showed $[35,45,46,51]$, the FoF MF obtained in cosmological simulations can be fitted by a function, $f(\sigma)$, of the variance of the linear density field $\sigma$. It has therefore been shown to be universal, independent of cosmology and redshift changes.

[35] claimed a universal behaviour of the mass function within 20\%. [36] showed the existence of deviations from universality and subsequent studies [e.g. 48, 52-54, 56] showed that the MF is not universal beyond the $5-10 \%$ level.

Further studies on the FoF MF showed evidences of non-universality in the time evolution $[52,53]$, or when changing the cosmology [54], while others showed an almost universal behaviour $[50] .^{3}$

[48] found a violation of universality at high redshift, due to the effective spectral index $n_{\text {eff }}$. Results of [47] were consistent with those of [48], but only at $z \leq 5 .{ }^{4}$ Their results exhibited $\mathrm{a} \simeq 5 \%$ residual in comparison to $z=0$, in agreement with the MF of [51]. The FoF MF of the Millennium simulation increased by $20 \%$ for the redshift range $z=0-10$ [48] and, when corrected for "spurious FoF linking between haloes", showed the same evolution (20\%) in the range $z=0-1[59]$.

Also [52] and [53] found deviations from universality even for the FoF MF and provided redshift-dependent fits. [53] proposed a redshift dependent FoF MF with an accuracy of $\simeq 2 \%$ at $z=0$ and an evolution up to $10 \%$ in the range $0<z<2,6 \times 10^{11}-3 \times 10^{15} M_{\odot}$. Similarly, [52] provided a fitting function that is accurate to $2 \%$ in the ranges $0 \lesssim z \lesssim$ 1 and $10^{10} \lesssim M \lesssim 10^{15.5} h^{-1} M_{\odot}$. [55] used the Millennium-XXL simulation to obtain a universal mass function accurate to $5 \%$ in the mass range studied. [54] found a universal behaviour of the FoF MF in the $\Lambda$ CDM model to a 5-10\% level. For different cosmologies they found deviations from the universal behaviour at $5 \%$ level. [50] calculated the MF from their

\footnotetext{
${ }^{3}$ According to [54], the MF changes with cosmology because of the change of the collapse threshold $\delta_{\mathrm{c}}$. Cosmologies having smaller values of $\delta_{\mathrm{c}}$ than that of the SCDM allow structures forming earlier with respect to the SCDM model, giving rise to a different halo MF. In order to have an exact universality, one needs a mechanism able to eliminate the effect of the past evolution of structure formation on the MF [54].

${ }^{4}$ They studied the mass function in the mass range $10^{7}<M<10^{13.5} h^{-1} M_{\odot}$.
} 
simulations using two different halo-finding methods: the FoF and the Spherical Overdensity (SO). The FoF MF was found weakly dependent on redshift and was well represented by a universal fit. A universal FoF MF fits to $10 \%$ the MF on their mass and redshift range. The MF universality across redshift was therefore valid only in some conditions and at a certain precision level. The SO MF had instead a redshift-dependent evolution. The proposed fit for the SO mass function was found valid within $\simeq 20 \%$ in the redshift range $0 \lesssim z \lesssim 20$, while the universal function fit was good within $\simeq 10 \%$.

Their result concerning the SO MF agreed qualitatively with [56], who found clearer evidences for deviations from universality in the case of the SO haloes with respect to FoF haloes. In their simulation the redshift dependence comes from the parameter $\Omega_{\mathrm{m}}$. Their fit to the MF at $z=0$ in the mass range $10^{11}<M<10^{15} h^{-1} M_{\odot}$ was valid within $5 \%$.

According to [49], the difference in behaviour, namely the almost universality of the FoF MF and non-universality of the SO MF, is related to the fact that FoF links structures before they merge to form a virialized halo. This produces an "inflation" of FoF masses, which, combined with the steep decrease of the MF, gives rise to an increase of the haloes number density.

In this paper, we will use the model of $[41-43,60]$ and $[61,62]$ to show the evolution of the mass function that can be obtained in a modified spherical collapse model, taking into account the proto-structures' angular momentum acquired by tidal interaction with neighbouring objects and dynamical friction [see also 63, 64].

In section 2 we find and discuss the barrier that is used in section 3 to determine the multiplicity and the mass function. Section 4 is devoted to the results, and section 5 to the discussion.

\section{ESF choices of barrier}

The semi-analytic model called extended Press-Schechter approach, based on stochastic processes, also known as "excursion set formalism" (ESF) [37, 39] is often used to model the halo formation statistics and mergers..$^{5}$

The halo statistics is obtained from the statistical properties of $\bar{\delta}\left(R_{\mathrm{f}}\right)$, the average overdensity within a window of radius $R_{\mathrm{f}}$. Density perturbations are represented by a Gaussian density field, smoothed with a filter (e.g., top-hat or $k$-space filters, the latter being a top-hat filter in momentum space). In a hierarchical universe, $\bar{\delta}\left(R_{\mathrm{f}}\right)$ vs $R_{\mathrm{f}}$ is a random walk [see 65]; in the excursion set approach, a halo forms when the random walk crosses a threshold value, or barrier, $\delta_{\mathrm{c}}$. Other quantities are often used (e.g., the mass variance $S$ ) instead of the filtering radius.

In this framework, the first key quantity, the probability that a random walk first crosses the threshold (barrier) between $S$ and $S+d S$, is called the first-crossing distribution. The last key quantity, known as multiplicity function, is related (as we will see in the following) to the mass function.

The Press-Schechter MF is re-obtained in the ESF by studying random walks and flat barriers. In order to improve the PS formalism, random walks with a non-flat barrier were considered. They are usually dubbed moving barrier as the barrier changes (moves) with $S$ or mass.

\footnotetext{
${ }^{5}$ The term "excursion set formalism", introduced by [37], comes from the name of the regions, excursion sets, upon which the determination of the mass function is based, in the stochastic processes theory, and characterized by a linear density contrast $\delta_{1}$ larger than the threshold $\delta_{\mathrm{c}}$.
} 
The first study on the subject was [41]. They found that the collapse threshold becomes mass dependent. In particular, they showed that in the set of objects that collapse at the same time, those less massive must have initially been denser than the more massive ones in order for the former to hold themselves together against stronger tidal forces. [42] and [43] showed the new threshold (barrier) to give rise to a MF in good agreement with observations.

The mass dependence in the barrier solves the problems of the PS MF, suppressing low mass haloes abundance and increasing it for massive ones (with respect to the PS MF) [66].

Such moving threshold is given by:

$$
\begin{aligned}
\delta_{\mathrm{cm}} & =\delta_{\mathrm{c}}(z)\left[1+\int_{r_{\mathrm{i}}}^{r_{\mathrm{ta}}} \frac{r_{\mathrm{ta}} L^{2} \cdot \mathrm{d} r}{G M^{3} r^{3}}\right] \\
& =\delta_{\mathrm{c}}(z)\left[1+\frac{8 G^{2}}{\Omega_{\mathrm{m}, 0}^{3} H_{0}^{6} r_{\mathrm{i}}^{10} \bar{\delta}(1+\bar{\delta})^{2}} \int_{r_{\mathrm{i}}}^{r_{\mathrm{ta}}} \frac{L^{2} \cdot \mathrm{d} a}{a^{3}}\right] \\
& =\delta_{\mathrm{c}}(z)\left(1+\frac{\beta}{\nu^{\alpha}}\right)
\end{aligned}
$$

where $\delta_{\mathrm{c}}(0) \simeq 1.686$ is the critical threshold for the spherical collapse in an Einstein-de Sitter (EdS) model, $r_{\text {ta }}$ is the turn-around radius, $r_{\mathrm{i}}$ the initial radius, $L$ the angular momentum acquired by the proto-structure, $a$ the expansion parameter, $H_{0}$ the Hubble constant and $\Omega_{\mathrm{m}, 0}$ the density parameter today. The values of the parameters are $\alpha=0.585$ and $\beta=0.46$ [e.g. $60,62]$. In eq. $(2.1)$, the quantity $\nu=\left(\frac{\delta_{\mathrm{c}}}{\sigma}\right)^{2}$ is used, where $\delta_{\mathrm{c}}$ is the critical overdensity needed for collapse in the spherical model and $\sigma(M)$ is the r.m.s. of density fluctuations on a comoving scale including a mass $M{ }^{6}$

In a later work, [44] used an ellipsoidal collapse, finding

$$
\delta_{\mathrm{ec}}=\delta_{\mathrm{c}}(z)\left[1+\beta_{1}\left(\frac{\sigma^{2}}{\delta_{\mathrm{c}}(z)^{2}}\right)^{\alpha_{1}}\right]=\delta_{\mathrm{c}}(z)\left(1+\frac{\beta_{1}}{\nu^{\alpha_{1}}}\right)
$$

with $\alpha_{1}=0.615$ and $\beta_{1}=0.485$.

To eqs. (2.1) and (2.2) correspond the two barriers

$$
B(M)=\sqrt{a} \delta_{\mathrm{c}}(z)\left(1+\frac{\beta}{a \nu^{\alpha}}\right)
$$

and

$$
B(M)_{\mathrm{ST}}=\sqrt{a_{1}} \delta_{\mathrm{c}}(z)\left(1+\frac{\beta_{1}}{a_{1} \nu^{\alpha_{1}}}\right),
$$

which give rise to a mass function in good agreement with simulations, when accurate values of $a$ are chosen, as shown by [62] and [44]. ${ }^{7}$

Changing the shape of the barrier (from flat to increasing with $S$ ) allows to incorporate several physical effects, from fragmentation and mergers [44], to the effects of tidal torques [41], cosmological constant [62] and dynamical friction (see the next sections).

As eqs. (2.3) and (2.4) show, the barrier found by [44], eq. (2.4) and [41, 42], eq. (2.3), are almost identical, as discussed in [68].

\footnotetext{
${ }^{6}$ Note that the usual definition of $\nu$ gives $\nu=\frac{\delta_{c}}{\sigma}$ [see 67]. The definition used in the text follows the ST notation.

${ }^{7}$ In the case of eq. (2.3), $a=0.67$ and for eq. (2.4), $a_{1}=0.707$.
} 


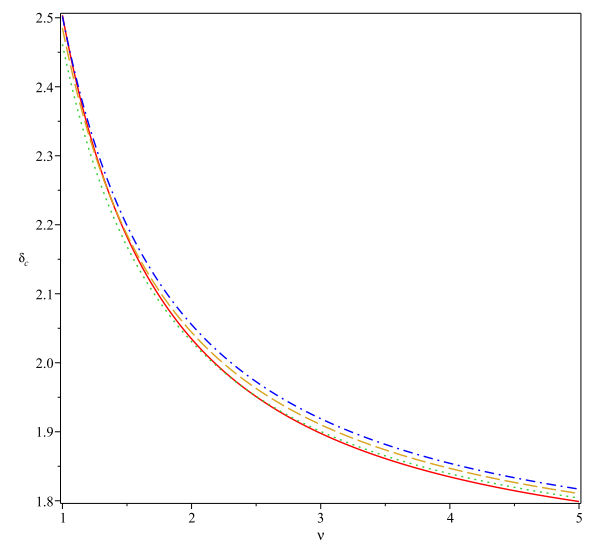

Figure 1. The collapse threshold $\delta_{\mathrm{c}}(\nu)$ as a function of $\nu$. The red solid line shows the result in [44], the green short-dashed line the results of [41], taking into account the effect of the tidal field, the orange long-dashed the result of [62] taking into account the effect of the tidal field and the cosmological constant, while the blue dot-dashed line takes into account the effect of the tidal field, the cosmological constant and the DF.

In the case of a $\Lambda \mathrm{CDM}$ model, the collapse threshold, calculated in [62], is given by

$$
\delta_{\mathrm{cm} 1}=\left[1+\int_{r_{\mathrm{i}}}^{r_{\mathrm{ta}}} \frac{r_{\mathrm{ta}} L^{2} \cdot \mathrm{d} r}{G M^{3} r^{3}}+\frac{\Lambda r_{t a}^{3}}{6 G M}\right]=\delta_{\mathrm{c}}(z)\left(1+\frac{\beta}{\nu^{\alpha}}+\frac{\Omega_{\Lambda} \beta_{2}}{\nu^{\alpha_{2}}}\right),
$$

where $\alpha_{2}=0.4, \beta_{2}=0.02$ and $\Omega_{\Lambda}$ is the contribution of the cosmological constant $\Lambda$ to the density parameter.

The barrier given by eq. (2.5), depending on the proto-structures' acquired angular momentum and on the cosmological constant, can be further improved by taking into account another important effect on the collapse of proto-structures: dynamical friction (hereafter $\mathrm{DF})$. This was done in [61], and is given by

$$
\begin{aligned}
\delta_{\mathrm{cm} 2} & =\delta_{\mathrm{co}}\left[1+\int_{r_{\mathrm{i}}}^{r_{\mathrm{ta}}} \frac{r_{\mathrm{ta}} L^{2} \cdot \mathrm{d} r}{G M^{3} r^{3}}+\Lambda \frac{r_{\mathrm{ta}} r^{2}}{6 G M}+\frac{\lambda_{o}}{1-\mu(\delta)}\right] \\
& \simeq \delta_{\mathrm{co}}\left[1+\frac{\beta}{\nu^{\alpha}}+\frac{\Omega_{\Lambda} \beta_{2}}{\nu^{\alpha_{2}}}+\frac{\beta_{3}}{\nu^{\alpha_{3}}}\right]
\end{aligned}
$$

where $\mu(\delta)$ is given in eq. (29) of [69] and appendix A, and $\lambda_{o}=\epsilon_{o} T_{c o}, \epsilon_{o}$ being proportional to the DF coefficient $\eta$ (see appendix A and eq. (23) of [70]) and $T_{c o}$ being the DF-less perturbation collapse time (see appendix A and eq. (24) of [70]). The angular momentum $L$ is calculated as shown in $[61,71,72]$ and in appendix A, while the DF term is obtained in [70] (see also appendix A).

Figure 1 compares $\delta_{\mathrm{c}}(\nu)$ obtained by [44] by means of an ellipsoidal collapse model with the modified collapse thresholds obtained by Del Popolo. In the plot, the solid line represents the result of [44], eq. (2.2), the dotted line eq. (2.1) obtained by [41], the dashed line eq. (2.5), namely the improvement of eq. (2.2) including $\Lambda$, and the dot-dashed line eq. (2.6), the improvement of eq. (2.5) accounting for the effect of DF.

All $\delta_{\mathrm{c}}(\nu)$ are monotonically decreasing functions of $\nu$ and mass $M$, and monotonically increasing functions of $S$, in contrast with other models [e.g. 73, 74]. Their behaviour tends to the typical value of the spherical collapse $\left(\delta_{\mathrm{c}} \simeq 1.686\right)$ for large $\nu$. This implies that less 
massive "peaks" (in the initial random field) form structures when crossing a higher threshold $\delta_{\mathrm{c}}$ than more massive ones.

Conversely, as high peaks are more probable in denser regions, the structure forming threshold $\delta_{\mathrm{c}}(M, z)$ is lower in overdense than underdense regions.

This reflects the different aspects of tides depending on the structures' mass. A peak acquires specific angular momentum $j$ proportionally to its turn-around time $t_{\mathrm{ta}}$, itself anticorrelated with the peak height: $j \propto t_{\mathrm{ta}} \propto \bar{\delta}(r, \nu)^{-3 / 2} \propto \nu^{-3 / 2}$. Hence smaller $\nu$ peaks are more sensitive to external tides, thus fixed time collapse leads them to be more overdense [41, $71,75-80]$. Low- $\nu$ peaks tend to resist more to gravitational collapse than high- $\nu$ peaks because they acquire larger $j$, and thus need higher density contrast to collapse and form structures. This is why structures need, on average, higher density contrast to collapse for small scales, where shear is more important.

Those results agree with $[77,78,81,82]$. The latter found out that larger shear and angular momentum slow down the collapse. As smaller scales statistically hold larger shear and angular momentum, collapse of structures at those scales require a higher density contrast. Those results have been extended more recently by [83-91] for models with dark matter and dark energy.

Angular momentum possesses similar effects as a non-zero cosmological constant as, in particular, it especially slows down large mass structures' collapse, however the effect of the cosmological constant vanishes at high redshift (see also section 4). DF adds similarly to the angular momentum and the cosmological constant effects, cumulating into the moving barrier behaviour that reduces small haloes' abundance, and increases that of massive haloes, with respect to a flat barrier (PS mass function). A similar behaviour characterises the ST model of ellipsoidal collapse, caused in this case by the larger ellipticity carried by smaller haloes, leading to a larger collapse time [see 92].

\section{Multiplicity and mass function}

In the ESF, the unconditional mass function $n(M, z)$, defined as the average comoving number density of haloes in a mass range $M-M+d M$ is [37]

$$
n(M, z)=\frac{\bar{\rho}}{M^{2}}\left|\frac{d \log \nu}{d \log M}\right| \nu f(\nu)
$$

where $\bar{\rho}$ is the background density. The quantity $f(\nu)$ is the so-called "multiplicity function", the distribution of the first crossing.

In the case of constant and linear barriers [68,93], one can obtain an analytical approximation for the first crossing. In other cases, the first crossing distribution can be obtained by generating a large ensemble of random walks. As shown by [68], for a large range of moving barriers, one can approximate the first crossing distribution using the formula

$$
f(S) d S=|T(S)| \exp \left(-\frac{B(S)^{2}}{2 S}\right) \frac{d S / S}{\sqrt{2 \pi S}},
$$

where $T(S)$ can be obtained using a Taylor expansion of $B(S)$ :

$$
T(S)=\sum_{n=0}^{5} \frac{(-S)^{n}}{n !} \frac{\partial^{n} B(S)}{\partial S^{n}}
$$


where $S \equiv S_{*}\left(\frac{\sigma}{\sigma_{*}}\right)^{2}=\frac{S_{*}}{\nu}$, being $\sigma_{*}=\sqrt{S_{*}}$. The multiplicity function is now given by $\nu f(\nu)=S f(S, t)$.

Applying the previous methods to the barrier given by eq. (2.4), the use of eqs. (3.2) and (3.3) gives, at fifth order $n=5$,

$$
\nu f(\nu)=\sqrt{a_{1} \nu / 2 \pi}\left[1+\beta_{1}\left(a_{1} \nu\right)^{-\alpha_{1}} g\left(\alpha_{1}\right)\right] \exp \left(-0.5 a_{1} \nu\left[1+\beta_{1}\left(a_{1} \nu\right)^{-\alpha_{1}}\right]^{2}\right),
$$

where

$$
g(\alpha)=\left|1-\alpha+\frac{\alpha(\alpha-1)}{2 !}-\ldots-\frac{\alpha(\alpha-1) \cdots(\alpha-4)}{5 !}\right|,
$$

and the values of $a_{1}, \alpha_{1}$ and $\beta_{1}$ have been given previously. Then [68] give

$$
\nu f(\nu) \simeq\left(1+\frac{0.094}{(a \nu)^{0.6}}\right) \sqrt{\frac{a \nu}{2 \pi}} \exp \left\{-\frac{1}{2} a \nu\left[1+\frac{0.5}{(a \nu)^{0.6}}\right]^{2}\right\}
$$

which is a good approximation to the first crossing distribution of the ellipsoidal barrier obtained through the simulations of unconstrained, independent random walks and that fitting the GIF simulations [see figure 2 of 68 ]

$$
\nu f(\nu) \simeq A\left(1+\frac{1}{(a \nu)^{0.3}}\right) \sqrt{\frac{2 a \nu}{\pi}} \exp (-a \nu / 2)
$$

where $A=0.322$.

The same method, for the barrier taking into account the cosmological constant, given by eq. (2.5), gives

$$
\begin{aligned}
\nu f(\nu) & =A_{1}\left(1+\frac{\beta g(\alpha)}{(a \nu)^{\alpha}}+\frac{\beta_{2} g\left(\alpha_{2}\right)}{(a \nu)^{\alpha_{2}}}\right) \sqrt{\frac{a \nu}{2 \pi}} \exp \left\{-\frac{1}{2} a \nu\left[1+\frac{\beta}{(a \nu)^{\alpha}}+\frac{\beta_{2}}{(a \nu)^{\alpha_{2}}}\right]^{2}\right\} \\
& \simeq A_{1}\left(1+\frac{0.1218}{(a \nu)^{0.585}}+\frac{0.0079}{(a \nu)^{0.4}}\right) \sqrt{\frac{a \nu}{2 \pi}} \exp \left\{-0.4019 a \nu\left[1+\frac{0.5526}{(a \nu)^{0.585}}+\frac{0.02}{(a \nu)^{0.4}}\right]^{2}\right\},
\end{aligned}
$$

The normalization factor $A_{1}=0.974$ must satisfy the constraint

$$
\int_{0}^{\infty} f(\nu) d \nu=1
$$

Finally, in the case of the barrier including, as in previous papers, the effects of angular momentum and $\Lambda$, and now introducing the new effect of Dynamical Friction, it is given by

$$
\begin{aligned}
\nu f(\nu) \simeq & A_{2} \sqrt{\frac{a \nu}{2 \pi}}\left(1+\frac{0.1218}{(a \nu)^{0.585}}+\frac{0.0079}{(a \nu)^{0.4}}+\frac{0.1}{(a \nu)^{0.45}}\right) \\
& \times \exp \left\{-0.4019 a \nu^{2.12}\left[1+\frac{0.5526}{(a \nu)^{0.585}}+\frac{0.02}{(a \nu)^{0.4}}+\frac{0.07}{(a \nu)^{0.45}}\right]^{2}\right\},
\end{aligned}
$$

where $A_{2}=0.93702$ and $a=0.707$. Thus eq. (3.10) presents our mass function, calculated in appendix B, whose validity shall now be confronted with simulations in the next section.

In the following we will use the CDM spectrum of [94, eq. (G3)].

At this point, it is important to stress that all numerical constants (except $a$ ) derive from barrier calculations: condition (3.9) gives the normalization constant A. Although 
the parameter $a$, which gives the number of high mass haloes, could also be obtained by the excursion set theory with a diffusing barrier, as shown by [95], it was determined as a fit to the massive haloes number in the simulations of [44], and thus could be interpreted as depending on the halo finder, which, in our case, similarly to [53], is a FoF with a linking length of $b=0.2$.

Since the number of halos, defined via FoF or SO techniques, is more affected by the change of technique at low masses rather than at high masses (provided the mass resolution of the simulation is good enough), the value of $a$ is largely unaffected by the choice of the specific algorithm. Different considerations hold instead for the dependence of $a$ as a function of the linking length parameter $b$. Given a background cosmological model, the virial overdensity $\Delta_{\text {vir }}$ can be evaluated, under certain assumptions, within the formalism of the spherical collapse model. Having this value and assuming for simplicity an isothermal profile for the dark matter halos, the mean density of the halo at the virial radius is $\rho_{R_{\mathrm{vir}}}=\bar{\rho} \Delta_{\mathrm{vir}} / 3$. Assuming that the density at the virial radius is represented by that of two particles in a sphere of radius $b$, one can relate it to a mean separation between particles. For an EdS model, $b=0.2$, while for a $\Lambda$ CDM cosmology with $\Omega_{\mathrm{m}}=0.25$ and $\Omega_{\Lambda}=0.75, b=0.156$. While the previously presented values proceed from the theoretical derivation of the linking length, in practice the value of an EdS model is also usually used for $\Lambda$ CDM cosmologies. As noted by [54], deviations from universality for the FoF mass function can be minimised by using the appropriate value of $\Delta_{\text {vir }}$, hence a correct value for $b$. Ref. [54] also quantified this and showed that the best choice for $b$ (called $b_{\text {univ }}$ ) can be obtained with the following relation:

$$
\left(\frac{b_{\text {univ }}}{0.2}\right)^{-3}=0.24\left(\frac{\Delta_{\text {vir }}}{178}\right)+0.68 \text {. }
$$

This empirical correlation can be explained in the light of the results of [96]. The authors showed that at $z=0$, the FoF overdensity for $b=0.2$ is significantly larger than 178 and that it depends on $b$ and on the halo concentration. Their analysis led to the following expression relating $b$ to $\Delta$ (the enclosed FoF overdensity)

$$
\left(\frac{b}{0.2}\right)^{-3}=\frac{\Delta+1}{244.86} \psi\left(c_{\Delta}\right),
$$

where

$$
\psi(c)=\frac{c^{2}}{\mu(c)(1+c)^{2}},
$$

and

$$
\mu(x)=\ln (1+x)-\frac{x}{1+x} .
$$

It is clear that the way the concentration parameter is defined will also affect the definition of the linking length. This implies therefore a dependence of the mass function on the linking length parameter $b$.

In the light of these considerations, it is reasonable to assume that using the appropriate linking length for a given cosmology, will leave $a$ unaffected. This could probably not be the case if, for a fixed cosmology, values of $b$ much different from the optimal one are used, due to the bridging problem affecting FoF methods. As also explained by [96], this issue needs to be investigated more deeply and quantitatively. 


\section{Results}

In the present section, we will compare our mass function at $z=0$ with several mass functions obtained from simulations over a large mass range. We will then compare its evolution with the result of [49], in the redshift range $0<z<10$ but over a more restricted mass range. Our choice was dictated by the lack of other exploitable simulations showing $z$-dependence: [46] is old, and [50] only plots some residuals and not the MF.

In the left panel of figure 2 , we plot the ratios of the mass function proposed by $[35,46$, $48,51-55,68]$ with [50], valid in the range $-0.55<\log \sigma^{-1}<1.31$ which at $z=0$ corresponds to the mass range $1.8 \times 10^{12}-7.0 \times 10^{15} h^{-1} M_{\odot} .[35,44,51,54,68]$ give a universal mass function, while $[48,52,53]$ provide an expression with z-dependent coefficients. ${ }^{8}$ Apart from older mass functions, like that of [46], our mass function agrees on average to a $\simeq 3 \%$ level in the mass range $10^{12}-10^{15} h^{-1} M_{\odot}$ with all the other mass functions. For $M>10^{15} h^{-1} M_{\odot}$ our MF agrees well with that of [53] (see the following). The latter MF also agrees with simulations data of more recent mass functions to better than $2 \%$ accuracy, while [55], based on the Millennium-XXL simulations, agrees to $5 \%$ with those simulations, [54] do so to $5-10 \%$, and [52] to $2 \%$. In the right panel of figure 2 , we plot the ratio between the Bhattacharya MF and ours. The discrepancy remains under $3 \%$ for the whole mass range $10^{12}-10^{16} h^{-1} M_{\odot}$.

In the panels of figure 3 and 4 , we plot the MF for different redshifts. The solid lines show our MF related to the multiplicity function given by eq. (3.10), while on the left panel, the dashed line displays the MF related to the multiplicity function (eq. (3.7)) of [68] and the diamonds with error-bars represent the MF obtained in the Bolshoi simulation by [49]. ${ }^{9}$ The MF redshift dependence comes from $\delta_{\mathrm{c}}(z)$. At $z=0$, the rightmost dashed curve, the mass function of [68], deviates from simulation data by less than $10 \%$ in the mass range $M_{\text {vir }}=5 \times 10^{9}-5 \times 10^{14} h^{-1} M_{\odot}$. At higher redshifts, [68] overpredicts simulation results, and that overprediction increases with redshift. At $z=6$, and for masses $M_{\mathrm{vir}}=$ $1-10 \times 10^{11} h^{-1} M_{\odot},[68]$ predicts 1.5 more halos than the simulation. The situation is much worse at $z=10$ since the MF of [68] predicts 10 times more haloes than the simulation. Our mass function (solid line) is in good agreement with the simulation with deviations $\lesssim 3 \%$, calculated by average on each curve from the central values, intersecting all the error bars.

In addition, as already reported, our result is also in agreement with simulations by [56] and [97]. In contrast, our result shows an increasing overprediction of the mass function of [68] going to larger masses and higher redshifts, together with a steepening of the MF with mass (there is a greater disagreement at larger masses). As stressed by [56], this behaviour reveals MF non-universality (i.e. dependence on redshift and cosmology), as the MF dependence with $z$ doesn't follow just the amplitude of $\sigma(M)$ (see definition in appendix B or [49]'s appendix B). Although our MF (eq. (3.10)) displays a universal-like structure, similarly to ST [68], its dependence on cosmology and $z$ differs from universal. This can be understood as follows.

The universality of the Sheth-Tormen (ST) formula [44, 45] proceeds from the multiplicity function only depending on the collapse threshold $\delta_{\mathrm{c}}$, a function both of $z$ and the cosmology. [54] stressed that the literature often neglects the cosmology dependence encoded in $\delta_{\mathrm{c}}$, because the Standard Cold Dark Matter (SCDM) scenario spherical collapse model, predicting $\delta_{\mathrm{c}}=1.686$ and $\Delta_{\mathrm{vir}}=178$ constant in redshift, has long been the structure formation studies' reference cosmology, and this $\delta_{\mathrm{c}}$ value has been long kept. It has also been shown

\footnotetext{
${ }^{8}$ The z-dependence in $[35,44,51,54,68]$ is parametrised through $\delta_{\mathrm{c}}(z)$.

${ }^{9}$ Note that the use of [68], now considered not particularly accurate, is shown for comparison with the work of [49] only, as they introduce a correction that performs more poorly than our MF, as seen below.
} 



Figure 2. Left panel: ratios between our mass function and others from literature, and the Watson MF at $z=0$ [50]. The red solid line represents the mass function proposed in this work; the blue short-dashed line the Sheth-Tormen mass function [68]; the purple dotted curve the Jenkins mass function [35]; the cyan short-dashed-dotted curve the Warren mass function [51]; the brown dashed and the pink dashed-dotted curves the two Reed mass functions [46, 48], respectively; the gray dashed-dotdotted curve the Angulo mass function [55]; the green and the violet solid curves show the Crocce [52] and the Bhattacharya [53] mass functions, respectively. Finally the light blue dashed-short-dashed curve represents the Courtin mass function [54]. Right panel: comparison between our proposed mass function and that of Bhattacharya [53] for different redshifts. We show the ratio of our mass function at a given redshift with respect to the one proposed by Bhattacharya, at $z=0$ for ensuring legible separation of the curves, in analogy to their figure 5. The red solid, green dashed and dotted blue lines show the ratio at $z=0, z=1$ and $z=2$, respectively. The corresponding data set points are those obtained in Bhattacharya's paper at $z=0, z=1$ and $z=2$, respectively.

that taking into account the cosmology dependence of $\delta_{\mathrm{c}}$ in non-standard cosmology provides a good agreement between analytic and numerical MF [26]. Alternatively, the mass function measured in numerical simulations has been directly fitted by multiplicity functions depending only on $\sigma[35,51,98]$, the resulting MF being thus manifestly independent of cosmological and redshift evolution, what is commonly understood as "universality" [cosmology and redshift independence of the relation between the linear and non-linear growth of structures; 99].

[54] showed a direct effect of evolution of $\delta_{\mathrm{c}}$, and $\Delta_{\text {vir }}$ with $z$ and cosmology on the MF, as haloes virialization, which depends upon cosmology and redshift, plays a role in determining it. These results demonstrate the importance of nonlinear effects in the MF prescription.

To summarize, the cosmology dependence of the non-linear collapse and virialization process creates deviations from universality, from cosmological model dependence of the spherical collapse threshold, responsible for deviations in the high-mass end of the MF, and accounting for it reduces MF discrepancy between models.

Ref. [49], preceded by several authors [e.g. 46], proposed an improvement to the ST mass function, multiplying it by a correction factor

$$
F(\delta)=\frac{(5.501 \delta)^{4}}{1+(5.500 \delta)^{4}},
$$




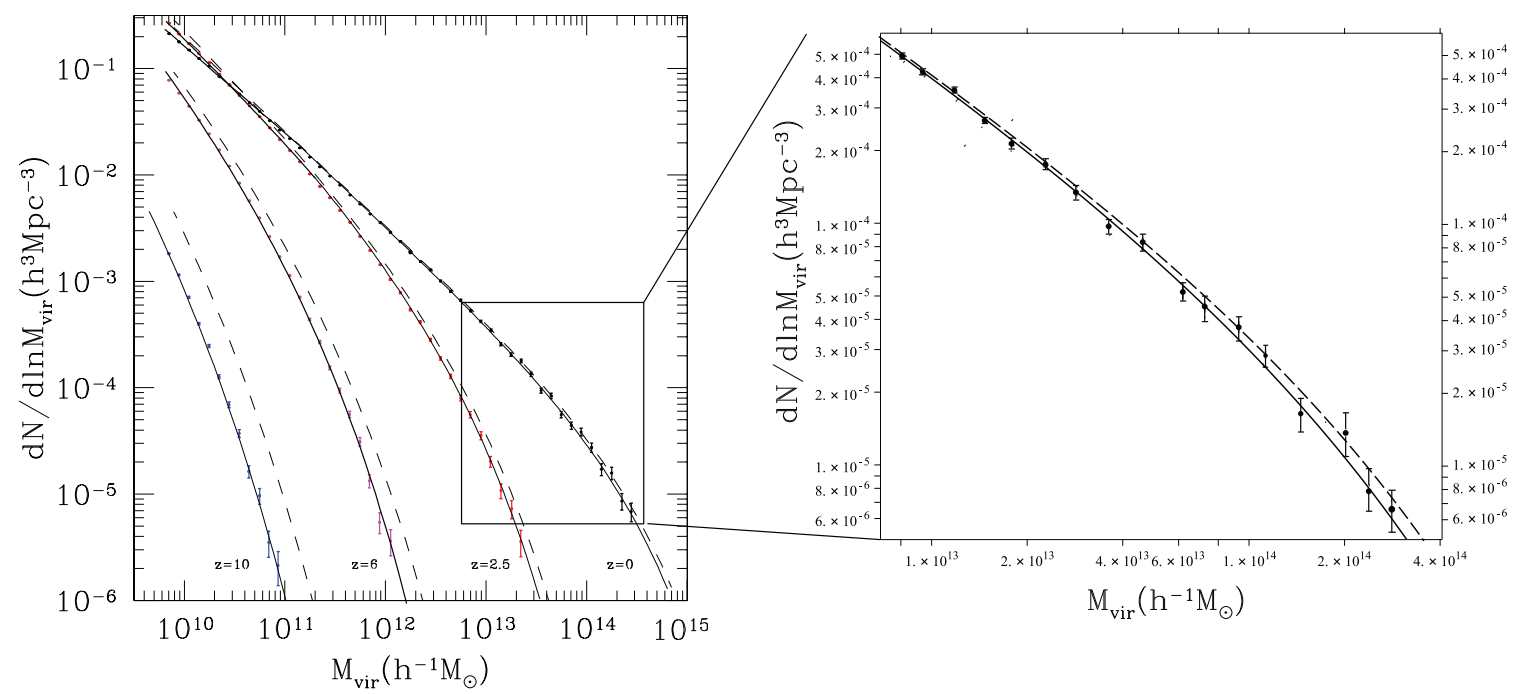

Figure 3. Comparison of the mass functions of [68] and this work with the Bolshoi MF. Left panel: the dashed line represents the ST MF, while the solid line ours. Diamonds represent the Bolshoi MF. From left to right $z$ receives the values $10,6,2.5,0$. Right panel: zoom of the $z=0 \mathrm{MF}$.

where $\delta$ is the linear growth factor normalized to unity today. [49] claimed that the corrected mass function deviates by less than $10 \%$ from simulations in the mass range $5 \times 10^{9}-5 \times$ $10^{14} h^{-1} M_{\odot}$. This is presented in figure 4, showing the comparison of the Bolshoi data (diamonds) with the correction by [49] (dashed line), and the result of our model (solid line). Note however that calculating the average error on $z=10$ yields $38 \%$ for the correction by [49] instead of the claimed $10 \%$, as opposed to our $3 \%$. It is evident there that our MF gives a much better result than the correction by [49].

The results displayed in figures 3 and 4 show that the MF generated from our barrier is in good agreement both with simulations at $z=0$ and with their redshift evolution with a precision of the order of $3 \%$. By contrast, the left panel of figure 3 shows the discrepancy between the predictions by [68] and simulations. [45] introduced the effects of asphericity considering an intuitive parametrization of an elliptical collapse without taking into account the interaction with neighbours (isolated spheroid), nor considering the effect of $\Lambda$ in the barrier. Thus, while their model is an improvement on the spherical collapse based PS model, it remains partial, leading to the aforementioned MF discrepancies with simulations. Our model, in contrast, takes into account the cosmological constant $\Lambda$, the effects of dynamical friction and of angular momentum, acquired through tidal torques. Such improvements give rise to a MF in good agreement with simulations, as we will detail in the following. Moreover, barriers increasing with $S$, like ours, allow mergers and fragmentation, whereas barriers decreasing with $S$ [e.g., 73, 74], are characterised by the facts that all walks cross them and fragmentation is not allowed.

In figure 5 we show the fractional accuracy of our proposed mass function with respect to the numerical mass function of [49] at $z=0$. The points and the error-bars correspond to the values from [49]. Note how all the error-bars intersect our curve. Errors are relatively small for low-mass objects and gradually increase towards higher masses. This is easily explained taking into account that the higher the mass, the lower is the number of objects. The point most differing from our mass function has a mass $M \approx 1.75 \times 10^{14} h^{-1} M_{\odot}$. Over 


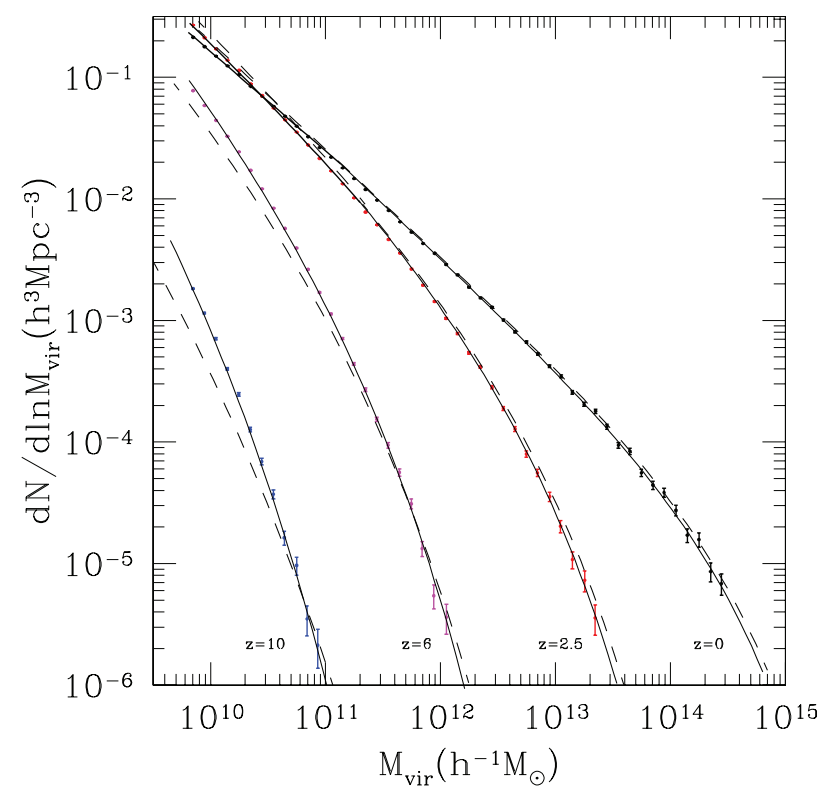

Figure 4. Comparison of our analytic mass function with the Bolshoi's of [49]. Curves and symbols are as in the left panel of figure 3, except that now the dashed line represents the correction by [49] to the ST MF.

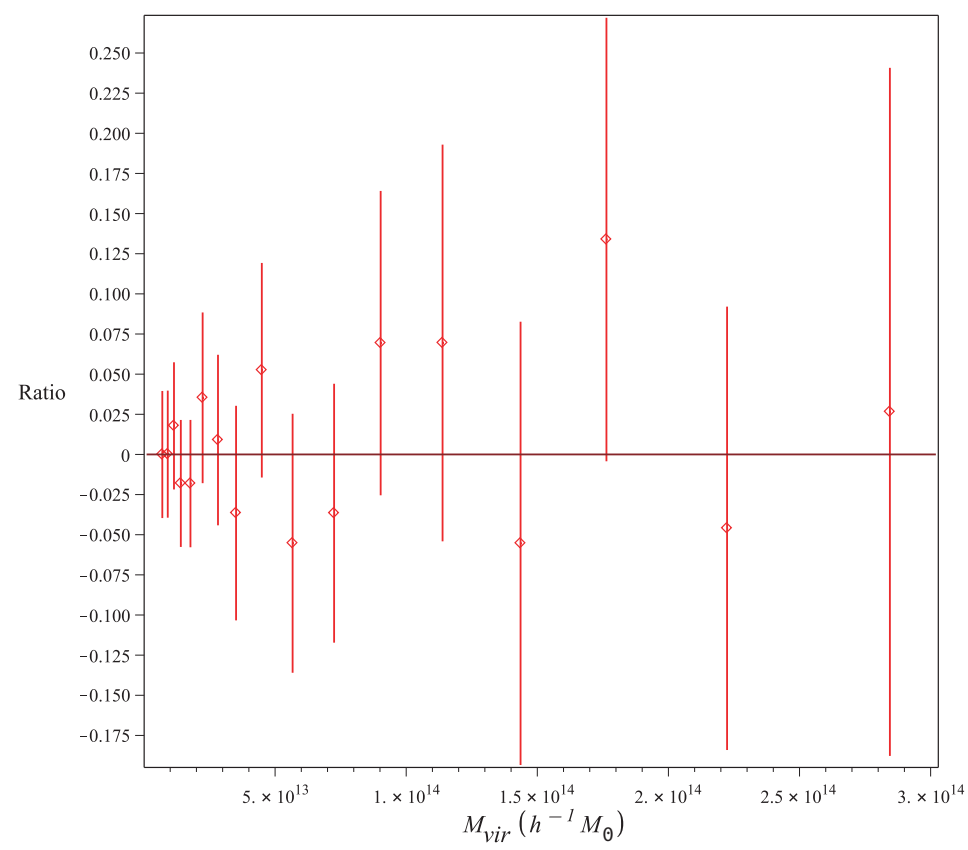

Figure 5. Fractional ratio between the numerical MF by [49] and our proposed mass function at $z=0$.

all the points, the accuracy of the mass function presented in this work with respect to the simulation used as comparison is, on average, better than $4 \%$.

It is also interesting to evaluate how the ratio between of our proposed mass function and that of Bhattacharya changes in time. This is shown in figure 2's right panel where we 


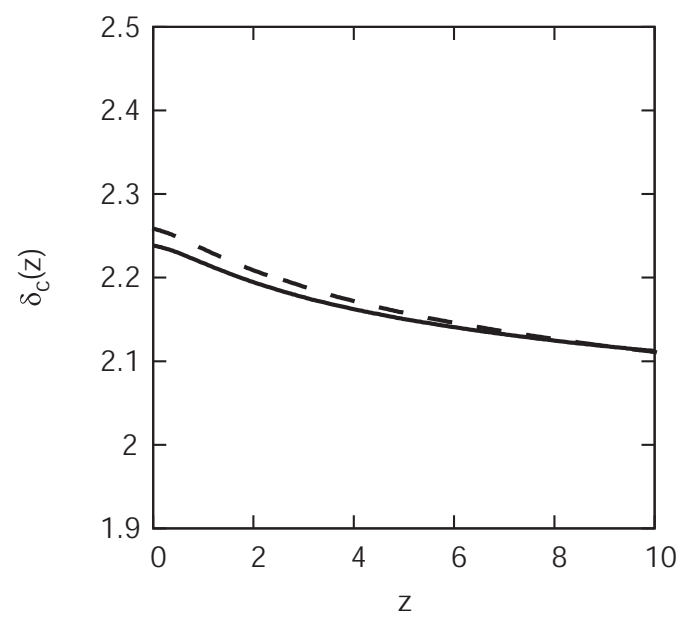

Figure 6. The collapse threshold in terms of $z$ when angular momentum and $\Lambda$ is taken into account (solid line) for a mass $M=10^{11} h^{-1} M_{\odot}$. The dashed line represents the same quantity when dynamical friction is taken into account.

consider the following redshifts: $z=0,1,2$. Points and corresponding error bars are those obtained in figure 5 of [53].

The data sets at $z=1$ and $z=2$ demonstrate that redshift evolution is important and must be taken into account; in addition, over the range of redshifts considered, the overall agreement is of the order of $3 \%$, except for few points at higher masses (low $\sigma$ ) where the agreement is at the $5 \%$ level.

Thus, a precise MF requires a precise determination of the barrier, whose shape depends on the effects of dynamical friction, the cosmological constant and angular momentum.

At this stage it is important to emphasize the need for a new and precise MF, especially when it agrees with previous ones [e.g., Bhattacharya's 53]. Multiplicity functions presented in this paper, such as Bhattacharya's and except for our's (eq. (3.10)), are produced by high resolution N-body simulations fits, similar in functional forms to [e.g. 35, 68]. These fits have no theoretical foundations, revealing the importance of obtaining a realistic analytical form from first principles. Such form is both able to better "describe" simulations and physically motivated. The MF (3.10) obtained in this paper does provide an excellent prediction of high resolution simulations, and at the same time derives from solid physical and theoretical arguments.

On top of this theoretical advantage, our approach can very accurately predict the dark matter halo distribution at much lower computational cost than high resolution simulations. This is because we can derive its functional form without having to rely on numerical results: it follows up directly by using an improved barrier.

In conclusion, the excursion set approach, with a structure formation physics motivated barrier, produces an excellent approximation to the numerical multiplicity function: improving the barrier form (with more and more physical effects: angular momentum acquisition, non-zero cosmological constant, etc.) increases the approximation accuracy. Moreover, this method displays a remarkable versatility: any effect, such as the presence of a non-zero cosmological constant, is very easy to take into account by embedding it in the barrier.

The role of angular momentum in shaping the MF was discussed in section 2, where we showed that it reduces or prevents structure formation, especially at small scales. 
In addition to the $\delta_{\mathrm{c}}$ dependence on mass, one needs to take into account its time evolution. In figure 6 we plot $\delta_{\mathrm{c}}(z)$, following [83] for a $\Lambda$ CDM model [for a generalization to DE models, see 84-86], taking into account the angular momentum and $\Lambda$ for a mass $M=10^{11} h^{-1} M_{\odot}$. The dashed line adds the effect of dynamical friction. Angular momentum causes $\delta_{\mathrm{c}}$ to be a monotonic decreasing function of $z$ while it already is the case for mass $M$ ): $\delta_{\mathrm{c}}(z)$ is larger than $\delta_{\mathrm{co}}$ at all values of $z$. Thus structure formation is "suppressed" at high $z$ by angular momentum. ${ }^{10}$ This explains why our MF predicts a smaller abundance than that of [68] with increasing $z$.

The effect of the cosmological constant can be understood from eq. (A.1) (discussed in detail in appendix A). The term involving the cosmological constant has the same effect as those involving angular momentum and DF, namely, slowing down the collapse [41, 42, 82, 100]. This gives rise to a delay in large-scale structure formation, reducing their abundance and steepening the MF. This would also produce a larger proportion of high- $z$ haloes, that would be smaller than the resolution of simulations [101]. At the same time the cosmological constant clearly decreases the number of halos in the high-mass tail, (Feyereisen private communication and paper in preparation).

As discussed by [53], since at high redshifts the effect of $\Lambda$ decreases, the non-universal evolution with redshift should be suppressed. However, despite the effect of $\Lambda$ reducing with $z$, the non-universal behaviour persists at high $z$ due to other factors [see 54].

Dynamical friction also slows down the collapse, similarly to the cosmological constant. Of the three effects taken into account, angular momentum is the strongest in slowing down the collapse, followed by dynamical friction. ${ }^{11}$

Before concluding, we want to point out that the agreement between our MF and the Bolshoi simulation data could be further improved assuming a slight redshift dependence of $A_{2}$ and $a$, as done in many of the papers cited in the Introduction.

\section{Discussion}

The determination of a high precision mass function is of fundamental importance. After the first improvements of the PS MF by [42] and [44, 45, 68], further advances came from N-body simulations, used as tools to calibrate proposed fitting formulas [e.g., 35, 46, 48, 50-54, 56] and more recently from a new diffusing barrier [95, 102-106].

Although MF obtained through simulations yield good approximations in several cases, the simulations black box nature, in which many physical effects are taken into account, makes it difficult to disentangle the role of those mechanisms in the shaping of the MF. In our approach, we selected physical effects known to play an important role in the shaping of the MF and explained with them why the PS MF gives bad fits to the observed MF, and why the ST MF has problems in reproducing it at high $z$. At the same time, the approach leaves us with a semi-analytical form of the MF in very good agreement with simulations. In this sense our result is much more physical than that of simulations and gives a fit to their results.

The paper shows that the introduction of a moving barrier makes the collapse threshold mass-dependent, contrary to the standard spherical collapse model, but in parallel with extended models where shear, tidal fields and/or angular momentum are taken into account.

\footnotetext{
${ }^{10} \mathrm{~A}$ similar result was obtained in [83, (figure 3$\left.)\right]$.

${ }^{11}$ For an alternative description of the effects of tidal shear and angular momentum for the $\Lambda$ CDM and dark energy models, we refer to [88-90].
} 
An interesting feature of a moving barrier is the possibility to introduce effects such as mergers, tidal torques, dynamical friction, and cosmological effects such as the cosmological constant (note that, as also pointed out by $[44,107]$ fitted their mass function with an EdS model).

The effect of introducing the cosmological constant remains minor compared with other effects such as tidal fields and angular momentum, but both the cosmological constant and the angular momentum slow down the collapse.

The positive consequence of these aspects is to solve the PS approach problems, in particular to reduce (increase) the number of objects at low (high) mass [62, 108]. A similar result has been found for the ellipsoidal collapse [44].

The barrier for the first crossing shapes the mass function and modify its functional form with respect to the simple PS formulation. The improved mass function yields results in very good agreement with $\mathrm{N}$-body simulations $[49,53]$, within $3 \%$ level at $z=0$ and during its time evolution (see figure 3). The general behaviour of our proposed mass function is in agreement with other functional forms proposed in literature, such as fits to numerical simulations (see figure 2) and shares with them, albeit at a lower level, the same problem: an excess of structures with respect to numerical simulations predictions at high redshifts.

\section{Acknowledgments}

The authors thank an anonymous referee for the useful comments that helped improving the scientific content of this work.

FP is funded by an STFC post-doctoral fellowship. The work of M.Le D. has been supported by PNPD/CAPES20132029. M.Le D. also wishes to acknowledge IFT/UNESP.

\section{A Inclusion of the angular momentum}

As already discussed, in hierarchical models, a perturbation collapses at a given time, when its overdensity exceeds a critical threshold. The barrier is the linear extrapolation of the threshold to the present time. We saw that [41] found a moving barrier, taking into account angular momentum acquisition. In $[61,62]$ the barrier was extended to account for the role of the cosmological constant and of DF.

The delay of collapse of a perturbation due to the acquisition of the angular momentum, the presence of dynamical friction, and a non-zero cosmological constant, can be obtained by solving the equation $[61,70,109-114]$ :

$$
\frac{d v_{r}}{d t}=\frac{L^{2}(r, \nu)}{M^{2} r^{3}}-g(r)-\eta \frac{d r}{d t}+\frac{\Lambda}{3} r,
$$

where $\Lambda$ is the cosmological constant, $g(r)$ the acceleration, $L(r, \nu)$ the angular momentum and $\eta$ the coefficient of dynamical friction. Recalling that the proper radius of a shell can be written as

$$
r\left(r_{\mathrm{i}}, t\right)=r_{\mathrm{i}} a\left(r_{\mathrm{i}}, t\right),
$$

where $a\left(r_{\mathrm{i}}, t\right)$ is the expansion parameter and $r_{\mathrm{i}}$ the initial radius, and that the mass is given by

$$
M=\frac{4 \pi}{3} \bar{\rho}\left(r_{\mathrm{i}}, t\right) a^{3}\left(r_{\mathrm{i}}, t\right) r_{\mathrm{i}}^{3},
$$


being the average density $\bar{\rho}\left(r_{\mathrm{i}}, t\right)=\frac{\bar{\rho}_{\mathrm{i}}\left(r_{\mathrm{i}}, t\right)}{a^{3}\left(r_{\mathrm{i}}, t\right)}=\frac{\bar{\rho}_{\mathrm{c}}(1+\bar{\delta})}{a^{3}\left(r_{\mathrm{i}}, t\right)}$ and $\rho_{\mathrm{ci}}=\frac{3 H_{\mathrm{i}}^{2}}{8 \pi G}$, eq. (A.1) may be written as

$$
\frac{d^{2} a}{d t^{2}}=-\frac{H^{2}(1+\bar{\delta})}{2 a^{2}}+\frac{4 G^{2} L^{2}}{H^{4}(1+\bar{\delta})^{2} r_{\mathrm{i}}^{10} a^{3}}-\eta \frac{d a}{d t}+\frac{\Lambda}{3} a .
$$

For $\eta=0$ the two equations become

$$
\frac{d v_{r}}{d t}=\frac{L^{2}(r, \nu)}{M^{2} r^{3}}-g(r)+\frac{\Lambda}{3} r
$$

and

$$
\frac{d^{2} a}{d t^{2}}=-\frac{H^{2}(1+\bar{\delta})}{2 a^{2}}+\frac{4 G^{2} L^{2}}{H^{4}(1+\bar{\delta})^{2} r_{\mathrm{i}}^{10} a^{3}}+\frac{\Lambda}{3} a
$$

Integrating eqs. (A.5) and (A.6) we can write

$$
\left(\frac{d r}{d t}\right)^{2}=\int \frac{2 L^{2}(r, \nu)}{M^{2} r^{3}} d r-2 g(r)+\frac{\Lambda}{3} r^{2}-2 C,
$$

and

$$
\left(\frac{d a}{d t}\right)^{2}=\frac{H_{\mathrm{i}}^{2}(1+\bar{\delta})}{a}+\int \frac{8 G^{2} L^{2}}{H_{\mathrm{i}}^{4}(1+\bar{\delta})^{2} r_{\mathrm{i}}^{10} a^{3}}+\frac{\Lambda}{3} a^{2}-2 C,
$$

where $C$ is the binding energy of the shell [see 112] and can be obtained using the conditions $d r / d t=0$ for eq. (A.7) and $d a / d t=0$ for eq. (A.8).

Integrating once more, recalling that $d r / d t=d a / d t=0$, we get [108]

$$
t_{\mathrm{ta}}=\int_{0}^{r_{\mathrm{ta}}} \frac{d r}{\sqrt{2\left[G M\left(\frac{1}{r}-\frac{1}{r_{\mathrm{ta}}}\right)+\int_{r_{\mathrm{ta}}}^{r} \frac{L^{2}}{M^{2} r^{3}} d r+\frac{\Lambda}{6}\left(r^{2}-r_{\mathrm{ta}}^{2}\right)\right]}},
$$

and $[42]$

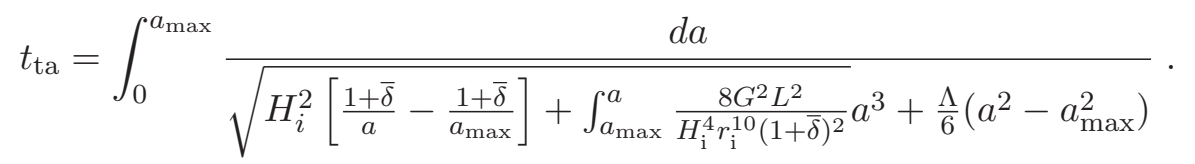

Using eqs. (A.7) and (A.9) (or eqs. (A.8) and (A.10)) it is possible to obtain the linear overdensity at turnaround and collapse $\left(\delta_{\mathrm{C}}\right)$, similarly to [112]. Solving numerically [113] eq. (A.9) for a given mass and turn-around time gives the turn-around radius, which is related to the binding energy through eq. (A.7) with $d r / d t=0$. The binding energy of a growing mode is uniquely given by the overdensity $\delta_{\mathrm{i}}$ at time $t_{\mathrm{i}}$. Linear theory can be used to get the overdensity at turn-around and collapse, $\delta_{\mathrm{c}}$. The connection between the binding energy, $C$, and $\delta_{\mathrm{i}}$ can be obtained by means of the relation $v-\delta_{\mathrm{i}}$ for the growing mode [e.g., $112,115]$.

As shown in [62], the threshold becomes

$$
\begin{aligned}
\delta_{\mathrm{c}} & =\delta_{\mathrm{co}}\left[1+\int_{r_{\mathrm{i}}}^{r_{\mathrm{ta}}} \frac{r_{\mathrm{ta}} L^{2} \cdot \mathrm{d} r}{G M^{3} r^{3}}+\Lambda \frac{r_{\mathrm{ta}} r^{2}}{6 G M}\right] \\
& \simeq \delta_{\mathrm{co}}\left[1+\frac{\beta}{\nu^{\alpha}}+\frac{\Omega_{\Lambda} \beta_{2}}{\nu^{\alpha_{2}}}\right]
\end{aligned}
$$


where the constants were already given in section 2 for eq. (2.5).

For $\eta \neq 0$, the barrier can be obtained similarly to the case $\eta=0$ starting from eq. (A.1) (or (A.4)).

In this case, the threshold becomes

$$
\begin{aligned}
\delta_{\mathrm{c}} & =\delta_{\mathrm{co}}\left[1+\int_{r_{\mathrm{i}}}^{r_{\mathrm{ta}}} \frac{r_{\mathrm{ta}} L^{2} \cdot \mathrm{d} r}{G M^{3} r^{3}}+\frac{\lambda_{o}}{1-\mu(\delta)}+\Lambda \frac{r_{\mathrm{ta}} r^{2}}{6 G M}\right] \\
& \simeq \delta_{\mathrm{co}}\left[1+\frac{\beta}{\nu^{\alpha}}+\frac{\Omega_{\Lambda} \beta_{2}}{\nu^{\alpha_{2}}}+\frac{\beta_{3}}{\nu^{\alpha_{3}}}\right]
\end{aligned}
$$

where $\alpha_{3}=0.07, \beta_{3}=0.45, \mu(\delta)=\frac{\sqrt{2} \pi}{3 c(\bar{\delta})}\left(\frac{1}{\bar{\delta}}+1\right)^{3 / 2}$ is given in [69] (eq. 29); $\lambda_{o}=\epsilon_{o} T_{c o}$, where

$$
\epsilon_{o}=\eta a^{3 / 2}=\frac{4.44\left[G m_{a} n_{a c}\right]^{1 / 2}}{N} \log \left(1.12 N^{2 / 3}\right),
$$

given $m_{a}$ and $n_{a}$, the mass and the number density of field particles (particles generating the fluctuating field), respectively, $N=\frac{4 \pi}{3} R_{\mathrm{sys}}^{3} n_{a}$ their total number, $n_{\mathrm{ac}}=n_{a} \times a^{3}$ their comoving number, and $R_{\text {sys }}$ the system radius [70], [42, appendix D], while $T_{c o}$ is the collapse time of a pure top hat model [116]

$$
T_{c 0}(r, \nu)=\frac{\pi}{H_{\mathrm{i}}[\bar{\delta}(r, \nu)]^{3 / 2}} .
$$

The angular momentum $L$, due to the tidal interaction with neighbours, is calculated getting the r.m.s. of the torque [see 76], [71, eq. (C5)], and then integrating the torque over time $[71,76$, eq. (35)] obtaining

$$
L(r, \nu)=\frac{1}{3}\left(\frac{3}{4}\right)^{2 / 3} \tau_{o} t_{0} \bar{\delta}_{o}^{-5 / 2} \int_{0}^{\pi} \frac{(1-\cos \vartheta)^{3}}{(\vartheta-\sin \vartheta)^{4 / 3}} \frac{f_{2}(\vartheta)}{f_{1}(\vartheta)-f_{2}(\vartheta) \frac{\delta_{o}}{\delta_{o}}} d \vartheta
$$

where $\tau_{o}$ is the tidal torque at $t_{o}$, and the functions $f_{1}(\vartheta)$ and $f_{2}(\vartheta)$ are given by [76], [see 71].

\section{B Expressions for the mass function}

In the following, we write the MF using the same notations and approximations of [49]. The mass function (eq. (3.1)) is given by

$$
n(M, z)=\frac{\bar{\rho}}{M^{2}} \frac{d \log \nu}{d \log M} \nu f(\nu) .
$$

Recalling that $d n=n d M$, that the background density $\bar{\rho}=\rho_{\mathrm{cr}} \Omega_{\mathrm{m}}$ and using the mass function of [35], $f(\sigma)=\frac{M}{\bar{\rho}} \frac{d M}{d \log \sigma^{-1}}$, eq. (B.1) can be written as

$$
\begin{aligned}
M \frac{d n}{d M} & =\bar{\rho} \frac{d \sigma(M)}{\sigma(M) d M} f(\sigma)=\Omega_{\mathrm{m}, 0} \rho_{\mathrm{cr}, 0} \frac{d \sigma(M)}{\sigma(M) d M} f(\sigma), \\
& =2.75 \times 10^{11}\left(h^{-1} \mathrm{Mpc}\right)^{-3} \Omega_{\mathrm{M}, 0} h^{2} M_{\odot} \frac{d \sigma}{\sigma d M} f(\sigma),
\end{aligned}
$$

where $\rho_{\mathrm{cr}, 0}$ is the critical density today ( $\bar{\rho}$ in eq. (3.1)), $M$ is the halo virial mass and

$$
\sigma^{2}(M)=\frac{\delta^{2}(a)}{2 \pi^{2}} \int_{0}^{\infty} k^{2} P(k) W^{2}(k, M) d k,
$$


is the mass variance, $P(k)$ the primordial power spectrum of perturbations, $W(k, M)$ the Fourier transform of the real-space top-hat window function. The linear growth-rate function $\delta(a)$ is given by

$$
\delta(a)=D(a) / D(1),
$$

where the expansion parameter is connected to redshift through $a=1 /(1+z)$. The growth rate factor $D(a)$ can be approximated, for $\Omega_{\mathrm{m}}>0.1$, using the expression proposed by $[111,117]^{12}$

Since we are going to compare our results to the Bolshoi simulation, we assume the approximation for $\sigma(M)$, the r.m.s. density fluctuation

$$
\sigma(M)=\frac{16.9 y^{0.41}}{1+1.102 y^{0.20}+6.22 y^{0.333}}, \quad y \equiv\left[\frac{M}{10^{12} h^{-1} M_{\odot}}\right]^{-1},
$$

whose accuracy is better than $2 \%$ for $M>10^{7} h^{-1} M_{\odot}$.

For the mass function of [68], we have

$$
f(\sigma)=A \sqrt{\frac{2 a_{1}}{\pi}}\left[1+\left(a_{1} \nu\right)^{-0.3}\right] \sqrt{\nu} \exp \left(-\frac{a_{1} \nu}{2}\right)
$$

where $\nu \equiv\left(\frac{1.686}{\sigma(M)}\right)^{2}, A=0.322$ and $a_{1}=0.707$.

For our barrier

$$
\begin{aligned}
\nu f(\nu) \simeq & A_{2}\left(1+\frac{0.1218}{(a \nu)^{0.585}}+\frac{0.0079}{(a \nu)^{0.4}}+\frac{0.1}{(a \nu)^{0.45}}\right) \sqrt{\frac{a \nu}{2 \pi}} \times \\
& \times \exp \left\{-0.4019 a \nu^{2.12}\left[1+\frac{0.5526}{(a \nu)^{0.585}}+\frac{0.02}{(a \nu)^{0.4}}+\frac{0.07}{(a \nu)^{0.45}}\right]^{2}\right\},
\end{aligned}
$$

where $A_{2}=0.965$.

\section{References}

[1] WMAP collaboration, D.N. Spergel et al., First year Wilkinson Microwave Anisotropy Probe (WMAP) observations: determination of cosmological parameters, Astrophys. J. Suppl. 148 (2003) 175 [astro-ph/0302209] [INSPIRE].

[2] A. Del Popolo, Dark matter and structure formation a review, Astron. Rep. 51 (2007) 169 [arXiv: 0801.1091] [INSPIRE].

[3] WMAP collaboration, E. Komatsu et al., Seven-year Wilkinson Microwave Anisotropy Probe (WMAP) observations: cosmological interpretation, Astrophys. J. Suppl. 192 (2011) 18 [arXiv: 1001.4538] [INSPIRE].

[4] A. Del Popolo, Non-baryonic dark matter in cosmology, AIP Conf. Proc. 1548 (2013) 2 [INSPIRE].

[5] K.T. Story et al., A measurement of the cosmic microwave background damping tail from the 2500-square-degree SPT-SZ survey, Astrophys. J. 779 (2013) 86 [arXiv:1210.7231] [INSPIRE].

\footnotetext{
${ }^{12}$ For $\Omega_{\mathrm{M}, 0}=0.27$ the error is smaller than $7 \times 10^{-4}$.
} 
[6] S. Das et al., The Atacama Cosmology Telescope: temperature and gravitational lensing power spectrum measurements from three seasons of data, JCAP 04 (2014) 014 [arXiv:1301.1037] [INSPIRE].

[7] A. Del Popolo, Nonbaryonic dark matter in cosmology, Int. J. Mod. Phys. D 23 (2014) 1430005 [arXiv:1305.0456] [InSPIRE].

[8] Planck collaboration, P.A.R. Ade et al., Planck 2015 results. XIII. Cosmological parameters, Astron. Astrophys. 594 (2016) A13 [arXiv: 1502.01589] [INSPIRE].

[9] S. Weinberg, The cosmological constant problem, Rev. Mod. Phys. 61 (1989) 1 [InSPIRE].

[10] A.V. Astashenok and A. del Popolo, Cosmological measure with volume averaging and the vacuum energy problem, Class. Quant. Grav. 29 (2012) 085014 [arXiv:1203.2290] [INSPIRE].

[11] R.A. Flores and J.R. Primack, Observational and theoretical constraints on singular dark matter halos, Astrophys. J. 427 (1994) L1 [astro-ph/9402004] [INSPIRE].

[12] V.F. Cardone and A. Del Popolo, Newtonian acceleration scales in spiral galaxies, Mon. Not. Roy. Astron. Soc. 427 (2012) 3176 [arXiv:1209.1524] [INSPIRE].

[13] A. Del Popolo, Density profile slope in dwarfs and environment, Mon. Not. Roy. Astron. Soc. 419 (2012) 971 [arXiv:1105.0090] [InSPIRE].

[14] A. Del Popolo, On the density profile slope of clusters of galaxies, Mon. Not. Roy. Astron. Soc. 424 (2012) 38 [arXiv:1204.4439] [InSPIRE].

[15] A. Del Popolo, V. Cardone and G. Belvedere, Surface density of dark matter haloes on galactic and cluster scales, Mon. Not. Roy. Astron. Soc. 429 (2013) 1080 [arXiv:1212.6797] [INSPIRE].

[16] A. Del Popolo and N. Hiotelis, Cusps and cores in the presence of galactic bulges, JCAP 01 (2014) 047 [arXiv:1401.6577] [INSPIRE].

[17] B. Moore, T.R. Quinn, F. Governato, J. Stadel and G. Lake, Cold collapse and the core catastrophe, Mon. Not. Roy. Astron. Soc. 310 (1999) 1147 [astro-ph/9903164] [INSPIRE].

[18] N. Hiotelis and A. Del Popolo, On the reliability of merger-trees and the mass growth histories of dark matter haloes, Astrophys. Space Sci. 301 (2006) 167 [astro-ph/0508531] [INSPIRE].

[19] N. Hiotelis and A. Del Popolo, Anomalous diffusion models for the formation of dark matter haloes, Mon. Not. Roy. Astron. Soc. 436 (2013) 163.

[20] G. Holder, Z. Haiman and J. Mohr, Constraints on $\Omega_{m}, \Omega_{\Lambda}$, and $\sigma_{8}$ from galaxy cluster redshift distributions, Astrophys. J. 560 (2001) L111 [astro-ph/0105396] [INSPIRE].

[21] Z. Haiman, J.J. Mohr and G.P. Holder, Constraints on quintessence from future galaxy cluster surveys, Astrophys. J. 553 (2000) 545 [astro-ph/0002336] [INSPIRE].

[22] J. Weller, R. Battye and R. Kneissl, Constraining dark energy with Sunyaev-Zeldovich cluster surveys, Phys. Rev. Lett. 88 (2002) 231301 [astro-ph/0110353] [INSPIRE].

[23] S. Majumdar and J.J. Mohr, Importance of cluster structural evolution in using X-ray and Sunyaev-Zeldovich effect galaxy cluster surveys to study dark energy, Astrophys. J. 585 (2003) 603 [astro-ph/0208002] [INSPIRE].

[24] F. Pace, J.-C. Waizmann and M. Bartelmann, Spherical collapse model in dark energy cosmologies, Mon. Not. Roy. Astron. Soc. 406 (2010) 1865 [arXiv:1005.0233] [InSPIRE].

[25] F. Pace, C. Fedeli, L. Moscardini and M. Bartelmann, Structure formation in cosmologies with oscillating dark energy, Mon. Not. Roy. Astron. Soc. 422 (2012) 1186 [arXiv:1111.1556] [INSPIRE]. 
[26] F. Pace, L. Moscardini, R. Crittenden, M. Bartelmann and V. Pettorino, A comparison of structure formation in minimally and non-minimally coupled quintessence models, Mon. Not. Roy. Astron. Soc. 437 (2014) 547 [arXiv:1307.7026] [InSPIRE].

[27] M. Malekjani, T. Naderi and F. Pace, Effects of ghost dark energy perturbations on the evolution of spherical overdensities, Mon. Not. Roy. Astron. Soc. 453 (2015) 4148 [arXiv: 1508.04697] [INSPIRE].

[28] T. Naderi, M. Malekjani and F. Pace, Evolution of spherical overdensities in holographic dark energy models, Mon. Not. Roy. Astron. Soc. 447 (2015) 1873 [arXiv:1411.7251] [INSPIRE].

[29] N. Nazari-Pooya, M. Malekjani, F. Pace and D. M.-Z. Jassur, Growth of spherical overdensities in scalar-tensor cosmologies, Mon. Not. Roy. Astron. Soc. 458 (2016) 3795 [arXiv: 1601.04593] [INSPIRE].

[30] S.R. Furlanetto, M. McQuinn and L. Hernquist, Characteristic scales during reionization, Mon. Not. Roy. Astron. Soc. 365 (2006) 115 [astro-ph/0507524] [INSPIRE].

[31] Z. Haiman and A. Loeb, What is the highest plausible redshift of luminous quasars?, Astrophys. J. 552 (2001) 459 [astro-ph/0011529] [INSPIRE].

[32] W.H. Press and P. Schechter, Formation of galaxies and clusters of galaxies by selfsimilar gravitational condensation, Astrophys. J. 187 (1974) 425 [INSPIRE].

[33] G. Efstathiou, C.S. Frenk, S.D.M. White and M. Davis, Gravitational clustering from scale free initial conditions, Mon. Not. Roy. Astron. Soc. 235 (1988) 715 [INSPIRE].

[34] M.A.K. Gross, R.S. Somerville, J.R. Primack, J. Holtzman and A. Klypin, CDM-variant cosmological models 1: simulations and preliminary comparisons, Mon. Not. Roy. Astron. Soc. 301 (1998) 81 [astro-ph/9712142] [INSPIRE].

[35] A. Jenkins et al., The mass function of dark matter halos, Mon. Not. Roy. Astron. Soc. 321 (2001) 372 [astro-ph/0005260] [INSPIRE].

[36] M.J. White, The mass function, Astrophys. J. Suppl. 143 (2002) 241 [astro-ph/0207185] [INSPIRE].

[37] J.R. Bond, S. Cole, G. Efstathiou and N. Kaiser, Excursion set mass functions for hierarchical Gaussian fluctuations, Astrophys. J. 379 (1991) 440 [INSPIRE].

[38] R.G. Bower, The evolution of groups of galaxies in the Press-Schechter formalism, Mon. Not. Roy. Astron. Soc. 248 (1991) 332 [INSPIRE].

[39] C.G. Lacey and S. Cole, Merger rates in hierarchical models of galaxy formation, Mon. Not. Roy. Astron. Soc. 262 (1993) 627 [INSPIRE].

[40] J.P. Gardner, Dependence of halo properties on interaction history, environment and cosmology, Astrophys. J. 557 (2001) 616 [astro-ph/0006342] [INSPIRE].

[41] A. Del Popolo and M. Gambera, Tidal torques and the clusters of galaxies evolution, Astron. Astrophys. 337 (1998) 96 [astro-ph/9802214] [INSPIRE].

[42] A. Del Popolo and M. Gambera, The effect of nonradial motions on the X-ray temperature distribution function and the two point correlation function of clusters, Astron. Astrophys. 344 (1999) 17 [astro-ph/9806044] [INSPIRE].

[43] A. Del Popolo and M. Gambera, Non radial motions and the shapes and the abundance of clusters of galaxies, Astron. Astrophys. 357 (2000) 809 [astro-ph/9909156] [INSPIRE].

[44] R.K. Sheth, H.J. Mo and G. Tormen, Ellipsoidal collapse and an improved model for the number and spatial distribution of dark matter haloes, Mon. Not. Roy. Astron. Soc. 323 (2001) 1 [astro-ph/9907024] [INSPIRE].

[45] R.K. Sheth and G. Tormen, Large scale bias and the peak background split, Mon. Not. Roy. Astron. Soc. 308 (1999) 119 [astro-ph/9901122] [INSPIRE]. 
[46] D. Reed et al., Evolution of the mass function of dark matter haloes, Mon. Not. Roy. Astron. Soc. 346 (2003) 565 [astro-ph/0301270] [INSPIRE].

[47] Z. Lukic, K. Heitmann, S. Habib, S. Bashinsky and P.M. Ricker, The halo mass function: high redshift evolution and universality, Astrophys. J. 671 (2007) 1160 [astro-ph/0702360] [INSPIRE].

[48] D. Reed, R. Bower, C. Frenk, A. Jenkins and T. Theuns, The halo mass function from the dark ages through the present day, Mon. Not. Roy. Astron. Soc. 374 (2007) 2 [astro-ph/0607150] [INSPIRE].

[49] A. Klypin, S. Trujillo-Gomez and J. Primack, Halos and galaxies in the standard cosmological model: results from the Bolshoi simulation, Astrophys. J. 740 (2011) 102 [arXiv:1002.3660] [INSPIRE].

[50] W.A. Watson, I.T. Iliev, A. D'Aloisio, A. Knebe, P.R. Shapiro and G. Yepes, The halo mass function through the cosmic ages, Mon. Not. Roy. Astron. Soc. 433 (2013) 1230 [arXiv: 1212.0095] [INSPIRE].

[51] M.S. Warren, K. Abazajian, D.E. Holz and L. Teodoro, Precision determination of the mass function of dark matter halos, Astrophys. J. 646 (2006) 881 [astro-ph/0506395] [INSPIRE].

[52] M. Crocce, P. Fosalba, F.J. Castander and E. Gaztanaga, Simulating the universe with MICE: the abundance of massive clusters, Mon. Not. Roy. Astron. Soc. 403 (2010) 1353 [arXiv: 0907.0019] [INSPIRE].

[53] S. Bhattacharya, K. Heitmann, M. White, Z. Lukic, C. Wagner and S. Habib, Mass function predictions beyond $\Lambda$ CDM, Astrophys. J. 732 (2011) 122 [arXiv: 1005.2239] [InSPIRE].

[54] J. Courtin, Y. Rasera, J.-M. Alimi, P.-S. Corasaniti, V. Boucher and A. Fuzfa, Imprints of dark energy on cosmic structure formation: II. Non-universality of the halo mass function, Mon. Not. Roy. Astron. Soc. 410 (2011) 1911 [arXiv: 1001.3425] [INSPIRE].

[55] R.E. Angulo, V. Springel, S.D.M. White, A. Jenkins, C.M. Baugh and C.S. Frenk, Scaling relations for galaxy clusters in the Millennium-XXL simulation, Mon. Not. Roy. Astron. Soc. 426 (2012) 2046 [arXiv:1203.3216] [inSPIRE].

[56] J.L. Tinker et al., Toward a halo mass function for precision cosmology: the limits of universality, Astrophys. J. 688 (2008) 709 [arXiv:0803.2706] [INSPIRE].

[57] M.J. White, The mass of a halo, Astron. Astrophys. 367 (2001) 27 [astro-ph/0011495] [INSPIRE].

[58] Z. Lukic, D. Reed, S. Habib and K. Heitmann, The structure of halos: implications for group and cluster cosmology, Astrophys. J. 692 (2009) 217 [arXiv:0803.3624] [INSPIRE].

[59] O. Fakhouri and C.-P. Ma, The nearly universal merger rate of dark matter haloes in $\Lambda C D M$ cosmology, Mon. Not. Roy. Astron. Soc. 386 (2008) 577 [arXiv:0710.4567] [INSPIRE].

[60] A. Del Popolo and M. Gambera, High velocity clumps and cluster of galaxies evolution, Baltic Astron. 11 (2001) 113 [astro-ph/0208341] [INSPIRE].

[61] A. Del Popolo, Some improvements to the spherical collapse model, Astron. Astrophys. 454 (2006) 17 [arXiv:0801.1086] [INSPIRE].

[62] A. Del Popolo, On the average comoving number density of halos, Astrophys. J. 637 (2006) 12 [astro-ph/0609100] [INSPIRE].

[63] V.F. Cardone, M.P. Leubner and A. Del Popolo, Spherical galaxy models as equilibrium configurations in nonextensive statistics, Mon. Not. Roy. Astron. Soc. 414 (2011) 2265 [arXiv:1102.3319] [INSPIRE]. 
[64] V.F. Cardone, A. Del Popolo, C. Tortora and N.R. Napolitano, Secondary infall model and dark matter scaling relations in intermediate redshift early-type galaxies, Mon. Not. Roy. Astron. Soc. 416 (2011) 1822 [arXiv:1106.0364] [inSPIRE].

[65] A. Del Popolo, On the cosmological mass function theory, Astron. Rep. 51 (2007) 709 [astro-ph/0609166] [INSPIRE].

[66] J. Zhang, C.-P. Ma and O. Fakhouri, Conditional mass functions and merger rates of dark matter halos in the ellipsoidal collapse model, Mon. Not. Roy. Astron. Soc. 387 (2008) L13 [arXiv:0801.3459] [INSPIRE].

[67] A. Del Popolo and M. Gambera, Peak mass in large-scale structure and dynamical friction, Astron. Astrophys. 308 (1996) 373.

[68] R.K. Sheth and G. Tormen, An excursion set model of hierarchical clustering: ellipsoidal collapse and the moving barrier, Mon. Not. Roy. Astron. Soc. 329 (2002) 61 [astro-ph/0105113] [INSPIRE].

[69] S. Colafrancesco, V. Antonuccio-Delogu and A. Del Popolo, On the dynamical origin of bias in clusters of galaxies, Astrophys. J. 455 (1995) 32 [astro-ph/9410093] [INSPIRE].

[70] V. Antonuccio-Delogu and S. Colafrancesco, Dynamical friction, secondary infall, and the evolution of clusters of galaxies, Astrophys. J. 427 (1994) 72.

[71] A. Del Popolo, The cusp/core problem and the secondary infall model, Astrophys. J. 698 (2009) 2093 [arXiv:0906.4447] [INSPIRE].

[72] A. Del Popolo and P. Kroupa, Density profiles of dark matter haloes on galactic and cluster scales, Astron. Astrophys. 502 (2009) 733 [arXiv:0906.1146] [INSPIRE].

[73] P. Monaco, A Lagrangian dynamical theory for the mass function of cosmic structures: 1. Dynamics, Mon. Not. Roy. Astron. Soc. 287 (1997) 753 [astro-ph/9606027] [INSPIRE].

[74] P. Monaco, A Lagrangian dynamical theory for the mass function of cosmic structures: 2. Statistics, Mon. Not. Roy. Astron. Soc. 290 (1997) 439 [astro-ph/9606029] [INSPIRE].

[75] Y. Hoffman, The dynamics of superclusters - the effect of shear, Astrophys. J. 308 (1986) 493.

[76] B.S. Ryden, Galaxy formation - the role of tidal torques and dissipational infall, Astrophys. J. 329 (1988) 589.

[77] P.J.E. Peebles, Previrialization, Astrophys. J. 365 (1990) 27.

[78] E. Audit, R. Teyssier and J.-M. Alimi, Nonlinear dynamics and mass function of cosmic structures: 1. Analytical results, Astron. Astrophys. 325 (1997) 439 [astro-ph/9704023] [INSPIRE].

[79] A. Del Popolo, On the universality of density profiles, Mon. Not. Roy. Astron. Soc. 408 (2010) 1808 [arXiv: 1012.4322] [INSPIRE].

[80] A. Del Popolo, Non-power law behavior of the radial profile of phase-space density of halos, JCAP 07 (2011) 014 [arXiv: 1112.4185] [INSPIRE].

[81] A. Del Popolo, E.N. Ercan and Z. Xia, Ellipsoidal collapse and previrialization, Astron. J. 122 (2001) 487 [astro-ph/0108080] [INSPIRE].

[82] A. Del Popolo, On the evolution of aspherical perturbations in the universe: an analytical model, Astron. Astrophys. 387 (2002) 759 [astro-ph/0202436] [INSPIRE].

[83] A. Del Popolo, F. Pace and J.A.S. Lima, Extended spherical collapse and the accelerating universe, Int. J. Mod. Phys. D 22 (2013) 1350038 [arXiv:1207.5789] [INSPIRE]. 
[84] A. Del Popolo, F. Pace and J.A.S. Lima, Spherical collapse model with shear and angular momentum in dark energy cosmologies, Mon. Not. Roy. Astron. Soc. 430 (2013) 628 [arXiv: 1212.5092] [INSPIRE].

[85] F. Pace, R.C. Batista and A. Del Popolo, Effects of shear and rotation on the spherical collapse model for clustering dark energy, Mon. Not. Roy. Astron. Soc. 445 (2014) 648 [arXiv: 1406.1448] [INSPIRE].

[86] A. Del Popolo, F. Pace, S.P. Maydanyuk, J.A.S. Lima and J.F. Jesus, Shear and rotation in Chaplygin cosmology, Phys. Rev. D 87 (2013) 043527 [arXiv: 1303.3628] [INSPIRE].

[87] A. Del Popolo, J.A.S. Lima, J.C. Fabris and D.C. Rodrigues, A unified solution to the small scale problems of the $\Lambda$ CDM model, JCAP 04 (2014) 021 [arXiv: 1404.3674] [INSPIRE].

[88] R. Reischke, F. Pace, S. Meyer and B.M. Schäfer, Spherical collapse of dark matter haloes in tidal gravitational fields, Mon. Not. Roy. Astron. Soc. 463 (2016) 429 [arXiv:1606.09207] [INSPIRE].

[89] R. Reischke, F. Pace, S. Meyer and B.M. Schäfer, Shear and vorticity in the spherical collapse of dark matter haloes, arXiv:1612.04275 [INSPIRE].

[90] F. Pace, R. Reischke, S. Meyer and B.M. Schäfer, Effects of tidal gravitational fields in clustering dark energy models, Mon. Not. Roy. Astron. Soc. 466 (2017) 1839 [arXiv:1612.03018] [INSPIRE].

[91] A. Del Popolo and M. Le Delliou, A unified solution to the small scale problems of the $\Lambda C D M$ model II: introducing parent-satellite interaction, JCAP 12 (2014) 051 [arXiv:1408.4893] [INSPIRE].

[92] J. Zhang, O. Fakhouri and C.-P. Ma, How to grow a healthy merger tree, Mon. Not. Roy. Astron. Soc. 389 (2008) 1521 [arXiv:0805.1230] [inSPIRE].

[93] R.K. Sheth, An excursion set model for the distribution of dark matter and dark matter halos, Mon. Not. Roy. Astron. Soc. 300 (1998) 1057 [astro-ph/9805319] [INSPIRE].

[94] J.M. Bardeen, J.R. Bond, N. Kaiser and A.S. Szalay, The statistics of peaks of Gaussian random fields, Astrophys. J. 304 (1986) 15 [INSPIRE].

[95] M. Maggiore and A. Riotto, The halo mass function from excursion set theory II. The diffusing barrier, Astrophys. J. 717 (2010) 515 [arXiv:0903.1250] [INSPIRE].

[96] S. More, A. Kravtsov, N. Dalal and S. Gottlober, The overdensity and masses of the friends-of-friends halos and universality of the halo mass function, Astrophys. J. Suppl. 195 (2011) 4 [arXiv:1103.0005] [INSPIRE].

[97] J.D. Cohn and M.J. White, Dark matter halo abundances, clustering and assembly histories at high redshift, Mon. Not. Roy. Astron. Soc. 385 (2008) 2025 [arXiv:0706.0208] [InSPIRE].

[98] E.V. Linder and A. Jenkins, Cosmic structure and dark energy, Mon. Not. Roy. Astron. Soc. 346 (2003) 573 [astro-ph/0305286] [INSPIRE].

[99] M.J. Francis, G.F. Lewis and E.V. Linder, Halo mass functions in early dark energy cosmologies, Mon. Not. Roy. Astron. Soc. 393 (2008) L31 [arXiv:0810.0039] [InSPIRE].

[100] A. Del Popolo and M. Gambera, Substructure effects on the collapse of density perturbations, Astron. Astrophys. 321 (1997) 691 [astro-ph/9610052] [INSPIRE].

[101] M.R. Feyereisen, S. Ando and S.K. Lee, Modelling the flux distribution function of the extragalactic gamma-ray background from dark matter annihilation, JCAP 09 (2015) 027 [arXiv: 1506.05118] [INSPIRE].

[102] M. Maggiore and A. Riotto, The halo mass function from excursion set theory I. Gaussian fluctuations with non-Markovian dependence on the smoothing scale, Astrophys. J. 711 (2010) 907 [arXiv:0903.1249] [INSPIRE]. 
[103] M. Maggiore and A. Riotto, The halo mass function from excursion set theory III. Non-Gaussian fluctuations, Astrophys. J. 717 (2010) 526 [arXiv:0903.1251] [INSPIRE].

[104] A. De Simone, M. Maggiore and A. Riotto, Excursion set theory for generic moving barriers and non-Gaussian initial conditions, Mon. Not. Roy. Astron. Soc. 412 (2011) 2587 [arXiv: 1007.1903] [INSPIRE].

[105] A. De Simone, M. Maggiore and A. Riotto, Conditional probabilities in the excursion set theory. Generic barriers and non-Gaussian initial conditions, Mon. Not. Roy. Astron. Soc. 418 (2011) 2403 [arXiv:1102.0046] [InSPIRE].

[106] C.-P. Ma, M. Maggiore, A. Riotto and J. Zhang, The bias and mass function of dark matter halos in non-Markovian extension of the excursion set theory, Mon. Not. Roy. Astron. Soc. 411 (2011) 2644 [arXiv:1007.4201] [InSPIRE].

[107] S. Murray, C. Power and A. Robotham, HMFcalc: an online tool for calculating dark matter halo mass functions, Astron. Comput. 3 (2013) 23 [arXiv:1306.6721] [INSPIRE].

[108] A. Del Popolo, A theoretical study of the mass temperature relation for clusters of galaxies, Mon. Not. Roy. Astron. Soc. 336 (2002) 81 [astro-ph/0205449] [INSPIRE].

[109] A. Kashlinsky, Dynamical friction in rotating systems - application to clusters and galaxies, Astrophys. J. 306 (1986) 374.

[110] A. Kashlinsky, Dynamical friction and evolution of the luminosity function in clusters of galaxies, Astrophys. J. 312 (1987) 497.

[111] O. Lahav, P.B. Lilje, J.R. Primack and M.J. Rees, Dynamical effects of the cosmological constant, Mon. Not. Roy. Astron. Soc. 251 (1991) 128 [INSPIRE].

[112] J.G. Bartlett and J. Silk, Galaxy clusters and the COBE result, Astrophys. J. 407 (1993) L45 [INSPIRE].

[113] E. Spedicato, E. Bodon, A. Del Popolo and N. Mahdavi-Amiri, ABS methods and ABSPACK for linear systems and optimization: a review, 4OR 1 (2003) 51.

[114] P.J.E. Peebles, Principles of physical cosmology, Princeton Univ. Pr., Princeton U.S.A., (1993) [INSPIRE].

[115] P.J.E. Peebles, The large-scale structure of the universe, Princeton Univ. Pr., Princeton U.S.A., (1980).

[116] J.E. Gunn and J.R. Gott, III, On the infall of matter into clusters of galaxies and some effects on their evolution, Astrophys. J. 176 (1972) 1 [INSPIRE].

[117] S.M. Carroll, W.H. Press and E.L. Turner, The cosmological constant, Ann. Rev. Astron. Astrophys. 30 (1992) 499 [INSPIRE]. 